\title{
Médias et violence durant la guerre du Golfe
}

\section{Arnaud Mercier}

\section{(2) OpenEdition \\ 12 Journals}

Édition électronique

URL : http://journals.openedition.org/conflits/296

DOI : 10.4000/conflits.296

ISSN : $1777-5345$

Éditeur :

CCLS - Centre d'études sur les conflits lilberté et sécurité, L'Harmattan

Édition imprimée

Date de publication : 15 mai 1993

ISSN : 1157-996X

Référence électronique

Arnaud Mercier, " Médias et violence durant la guerre du Golfe », Cultures \& Conflits [En ligne], 09-10 |

printemps-été 1993, mis en ligne le 04 mars 2005, consulté le 30 mars 2021. URL : http://

journals.openedition.org/conflits/296; DOI : https://doi.org/10.4000/conflits.296

Ce document a été généré automatiquement le 30 mars 2021.

Creative Commons License 


\title{
Médias et violence durant la guerre du Golfe
}

\author{
Arnaud Mercier
}

1 L'analyse qui a guidé le comportement des acteurs de cette guerre est que la violence, les images de douleur et de mort, sont devenues insupportables dans les sociétés occidentales. Il faut donc les cacher pour ne pas occasionner dans l'opinion un choc émotionnel propre à contrarier la conduite des objectifs politiques de l'Etat, passant ici par la guerre. Les militaires et les pouvoirs politiques ont donc mis en place un système de censure, afin d'empêcher les journalistes de donner à voir des images de mort comme celles de la Guerre du Vietnam ${ }^{1}$. Il y a eu en quelque sorte violence des censeurs pour censurer la violence. Cependant, cette censure n'est pas la seule variable capable d'expliquer le mode de présentation de la violence dans les médias. En effet, celle-ci n'a pas totalement été masquée; elle a plutôt été retravaillée, représentée, en passant à travers le prisme télévisuel et journalistique. La violence a été montrée mais elle fut euphémisée, mise en scène, simulée, voire esthétisée. Il convient donc de mettre au jour ces logiques de traitement de la violence, tout en déterminant ce qui relève d'une stratégie délibérée des censeurs et ce qui tient aux logiques médiatiques. Pour ce faire, notre corpus sera composé des journaux télévisés des trois grandes chaînes nationales françaises de l'époque, ainsi que des représentations photographiques proposées dans les trois numéros de la revue "Edition Spéciale", dans le numéro hors série de VSD, et dans les magazines américains Newsweek et Time. Ce travail accompli, nous aimerions pour conclure élargir notre propos en nous interrogeant sur les stratégies des acteurs nationaux quant à l'utilisation qui est faite d'un outil transnational comme la télévision. En effet, la gestion de la violence dans le cadre d'une communication politique nationale est désormais problématique face à un univers médiatique mondialisé 2 .

\section{LA VIOLENCE DES CENSEURS}


3

Les militaires ont organisé le système dit des "pools" pour contenir l'activité des journalistes et notamment des cameramen. Cette invention américaine a pour but d'intégrer un petit groupe de journalistes aux opérations militaires (mais surtout dans les troupes d'arrière garde!) qui partagent ensuite anonymement leurs informations avec les reporters restés à l'arrière. L'état-major peut ainsi concilier une double exigence : garantir la sécurité des journalistes et contrôler l'information. Les "pools" sont d'une part une réaction à la relative liberté laissée lors de la guerre du Vietnam en matière de couverture médiatique et, d'autre part, une réponse aux nombreuses critiques que suscita l'absence de tout journaliste lors des premières heures de l'intervention à la Grenade. Le pool est un moyen terme, jugé tout à fait satisfaisant par les militaires, et considéré comme un moindre mal par les journalistes. Il fut testé pour la première fois lors de l'opération au Panama et, devant le peu de critiques qu'il suscita de la part des médias américains, il fut repris dans le Golfe avec plus de rigueur. Les militaires comprirent que leurs objectifs ne pourraient être atteints s'ils apparaissaient, dans leur pratique, comme trop en porte-à-faux par rapport aux valeurs démocratiques. Le travail de légitimation des buts de la guerre, passant par l'affirmation de la nécessité de faire respecter le Droit International ainsi que le rôle et la mission de l'ONU, aurait été partiellement anéanti si des témoignages étaient venus montrer la cruauté de la guerre. Les autorités militaires ont donc "négocié" avec les journalistes les modalités de la censure. Ils tentèrent d'obtenir de cette manière un simulacre d'accord démocratique, un compromis au nom de la préservation des intérêts bien compris de chacun. Pour ce faire, ils ont utilisé des arguments de légitimation recourant à des valeurs largement partagées. Le bon sens : il faut pouvoir protéger les journalistes. Le patriotisme : il faut protéger la vie des soldats, et ne pas affaiblir les possibilités de victoire, en informant l'ennemi des mouvements de troupes. La morale et la pudeur : les familles des victimes doivent être averties d'abord, et ne pas apprendre par la presse la mort de l'un des leurs. La guerre ne doit pas être l'occasion d'un voyeurisme malsain. Il s'agit en quelque sorte d'une argumentation destinée à mettre les journalistes dans une position de coupables et d'inconscients aux yeux du public s'ils n'acceptent pas les restrictions imposées par l'armée au libre exercice de leur métier. Les militaires font appel à l'opinion pour placer les journalistes face à un choix inacceptable pour eux: se soumettre où être réduits au silence. L'obligation de signer une charte de bonne conduite, l'affirmation par l'armée de sa volonté de coopérer, et le recours à un argumentaire qui fait écho dans l'opinion, sont les vecteurs de cette violence excommunicationnelle. Il faut préciser que l'exercice direct de la censure paraît désormais incongru dans les sociétés démocratiques occidentales où les médias ont acquis une légitimité et une place sans équivalent dans l'histoire. Les militaires ont donc renoncé à une autorité coercitive aliénante impliquant le recours à la violence physique concrète (fermeture de locaux de presse, expulsions ou arrestations de journalistes), au profit d'une autorité normative jouant sur l'appel à la discipline et même à un certain degré de participation, tout en prétendant s'insérer dans les logiques de la communication. La discipline reposait sur un échange entre l'obéissance et des images, la participation joue sur le registre du patriotisme et du bon sens. D'ailleurs les journalistes se sont eux-mêmes livrés au jeu de la légitimation du recours à la guerre, par la stigmatisation de l'adversaire. Le système mis en place par les militaires, et en partie intériorisé par les journalistes, avait pour objectif de maîtriser les réactions d'émotion du public, face à des images porteuses de violence. La guerre du Vietnam fut en effet traumatique pour nombre de 
citoyens américains, car la guerre nécessite clairement l'abandon, au nom des fins poursuivies, d'un certain nombre d'idéaux démocratiques ${ }^{3}$. L'exécution sommaire d'un Vietcong d'une balle dans la tête reste une des images les plus célèbres, (avec celle de la fillette qui court, brûlée au napalm) et l'une de celles qui provoqua le plus "d'indignation" (au sens premier de sentiment de colère que soulève une action révoltant en nous la conscience morale ou le sens de la justice). Eviter que des forces politiques n'utilisent ce ressort émotionnel et cet état de dissonance cognitive ${ }^{4}$ contre les objectifs politiques et militaires des gouvernants, est donc la priorité des gouvernements démocratiques dans toute situation d'utilisation de la violence armée ${ }^{5}$. Comme le prouvent les différents black-out médiatiques durant les interventions à la Grenade, aux Malouines ou au Tchad ${ }^{6}$. En effet, la construction du système étatique caractérisé par le monopole de la violence physique légitime, est le fruit d'un processus de civilisation, de domestication des pulsions et d'avènement de l'autocontrôle que décrit Norbert Elias. L'Etat se légitime en exigeant un autocontrôle et non en imposant un contrôle coercitif, tout en garantissant en échange la sécurité de chacun. Les institutions sociales trouvent leur énergie et leur justification dans la quantité d'agressivité qu'elles empruntent aux individus qu'elles contrôlent. Cette monopolisation est le fruit d'un contrat rappelant celui du Léviathan, mais qui stipule explicitement dans le cas des sociétés démocratiques que cette énergie confisquée sera "employée de manière productive, raisonnable, altruiste et, si possible, sans violence"7. Cette domestication de la violence, une fois intériorisée, rend donc insoutenable la soumission à un spectacle violent et difficilement légitimable le recours aux horreurs de la guerre. L'appel à la force peut donc plus facilement - pour peu que les images de violence soient retravaillées socialement - occasionner une contestation, comme les manifestations américaines contre la guerre du Vietnam l'ont montré. Enfin, une guerre menée au nom de la justice et d'une juste cause, serait également apparue comme beaucoup plus contestable si la censure - et souvent la désinformation - n'était venue masquer la disproportion des forces en présence. La censure avait pour but d'éviter de soulever les opinions publiques arabes et "d'indigner" les opinions publiques occidentales, au nom d'une injustice flagrante. Car la médiatisation d'un événement finit par interagir avec l'action, comme le souligne Daniel Hermant: "Dès qu'un événement atteint une certaine ampleur, il engendre un flux d'images et de représentations qui, ensuite, est réinjecté dans le processus lui-même et interfère dans son évolution"s. Il est donc de l'intérêt des acteurs de maîtriser les flux des représentations de la guerre.

\section{UNE GUERRE SANS VIOLENCE}

5 La couverture médiatique de la guerre du Golfe a été marquée par la transfiguration de la violence martiale. Plusieurs procédés ont été mis en oeuvre pour arriver à maitriser l'impact auprès du public des images de la guerre. Il ne s'agissait nullement d'interdire les diffusions de toutes images violentes mais de les canaliser. Les images de violence portent en elles deux sentiments contradictoires que sont la répulsion et la fascination9. Les autorités politico-militaires ont donc tenté de limiter les manifestations possibles de répulsion et ont joué le plus souvent du registre de la fascination. . L'euphémisation. Les seules images de destruction diffusées furent celles 
des images vidéo d'avions alliés envoyant, au centimètre près, des missiles sur "un objectif stratégique" (pont, usine, caserne, aéroport..), là même où des civils ne devraient donc pas être. Dans le cas contraire, les militaires s'en excusent, et parlent alors de "dégâts collatéraux". Ce terme même, relayé par les journalistes, est le meilleur symbole du processus d'euphémisation à l'oeuvre: il ne s'agit pas de destruction mais uniquement de dégâts (expression qui rappelle une tournure journalistique classique à propos d'un attentat ou d'une légère explosion de gaz: "quelques dégâts matériels, mais pas de blessés"), et ces dégâts sont collatéraux, sousentendu involontaires, mais quand même nécessaires à cette guerre, puisque l'important était de détruire la cible, ce qui était "central" par rapport au "collatéral". Comme l'écrivait Frédéric Bon: "La guerre, sauf rares exceptions, n'ose plus se présenter dans le monde contemporain sous son véritable nom" ${ }^{10}$. . L'esthétisation. Autre modalité de l'appel au pouvoir fascinant des images violentes. Le bombardement de Bagdad des premières nuits, avec ses balles traçantes dans une luminosité verdâtre, était comparé à un feu d'artifice, par les commentateurs de CNN. Un journaliste de FR3 par exemple tint les propos suivants : "Bagdad illuminée par les serpentins que forment les balles traçantes". De même les journalistes n'ont cessé de faire part de leur émerveillement face à l'affrontement nocturne et en direct entre missiles Scud et Patriot, ou encore face aux images prises par les avions alliés, les comparant à celles que l'on peut voir dans les cockpits de Formule 1. Les journalistes eux-mêmes furent fascinés par ces images. Les présentateurs de l'émission spéciale de TF1 au premier jour de l'offensive (le 18 janvier 1991) en témoignent. Michelle Cotta affirme : "Les photos aériennes de bombardement au laser diffusées par l'armée américaine sont extraordinaires". "Ce sont effectivement des images tout à fait étonnantes" rajoute Gérard Carreyrou. "Dans quelques instants, d'autres images tout à fait étonnantes"11. . La technologisation. Les alliés ont mis en avant la maitrise technique et technologique de leur logistique et de leur armement. Les reportages photographiques et télévisés se sont tous longuement étendus sur la puissance de feu réunie et l'efficacité de l'organisation américaine. Les journalistes développèrent l'idée de la "première guerre high-tech, une guerre de troisième type où la technologie devrait faire la décision". Cette guerre était avant tout présentée comme "le plus formidable déploiement de troupes et de matériel depuis la Seconde Guerre mondiale"12. La guerre devient donc avant tout une simple maitrise technologique d'outils de destruction "intelligents" et "propres", c'est-à-dire censés respecter les populations civiles. Cette fascination pour la technologie militaire rencontre les logiques journalistiques. En effet, les rédactions du monde entier ont également déployé des efforts financiers considérables pour bénéficier de tous les acquis des technologies de la communication, la guerre du Golfe ayant été placée sous le signe du direct absolu et des multiplex planétaires. Gérard Carreyrou explique cette fascination comme pour s'en excuser: "Bien sûr, certains disent qu'on jubile à l'antenne, qu'on semble y prendre du plaisir. Je ne dis pas que c'est bien. Je dis que c'était inévitable parce qu'enfin - si j'ose dire - le système qu'on avait mis en place depuis des mois allait pouvoir fonctionner"13. Ce système devait permettre de réaliser l'un des fantasmes de l'humanité: celui du panoptique ${ }^{14}$, de l'être omniscient qui "grâce aux satellites, aux avions espions ou furtifs, saurait tout"15. . La distanciation. Différents procédés sont utilisables pour produire cet effet par rapport au réel. Il y eut d'abord la déréalisation qui fait appel au registre ludique, renforçant encore le pouvoir fascinant des images diffusées. Les seules images de bataille qui aient été montrées sont celles d'une violence médiatisée (au sens étymologique du terme). 
Les journaux et la télévision ont proposé des animations graphiques des batailles à venir ou en train de se dérouler. Les soldats, les individus concrets n'étaient représentés que par des carrés en carton symbolisant un uniforme, à côté de carrés identiques représentant des avions, des hélicoptères ou des chars. Les images de destruction diffusées avaient été prises depuis les avions, leur qualité technique, le caractère noir et blanc et l'angle de vue très resserré ont suffi à affaiblir leur qualité violente, avec une référence évidente aux jeux vidéo qui leur a donné une dimension ludique prononcée. De la même façon, les télévisions se sont livrées à un travail de simulation. La plupart des images de guerre ont été tirées des archives, et notamment de la guerre 14-18, pour illustrer les menaces de guerre chimique. Ces images véhiculaient en fait une angoisse sourde. Leur caractère historique, la mise à distance que les images contiennent (noir et blanc, grains, sauts d'images, vitesse de déroulement de la pellicule) suffisent au téléspectateur pour qu'il ne se sente pas totalement concerné, mais inquiet tout de même. Enfin, les médias ont suivi une logique de désincarnation. De très long reportages ont été consacrés aux carcasses calcinées des milliers de véhicules en tout genre qui jonchaient la route Bagdad-Koweit, peu après la libération. Ces images d'une rare violence laissent imaginer le carnage qui s'est produit. Pourtant les images ne comprennent aucun cadavre, l'armée n'ayant emmené les journalistes sur ce champ de bataille qu'une fois "nettoyé" des corps qui s'y trouvaient. De même les seules images de la première bataille, celle de Khafji, sont celles de carcasses de blindés en feu et de soldats alliés patrouillant dans une ville déserte, avec tout au plus un cadavre isolé. Le travail de désincarnation s'est poursuivi par le silence maintenu sur le nombre des victimes. Comme si la guerre, peu coûteuse du côté allié, n'avait pas fait non plus de morts du côté irakien. Le flou le plus intense a été entretenu autour du tableau chiffré des victimes. Les journalistes s'en désolent: "Les avions décollent bien, ils reviennent bien, l'armée nous présente parfois un raid, le plus spectaculaire, mais de bilan global des opérations et des dégâts irakiens, il n'y a rien, désespérément rien !" ${ }^{16}$. L'incertitude sur les statistiques a d'ailleurs été justifiée avec franchise par le Pentagone, qui se déclara incapable d'évaluer le nombre de soldats irakiens à avoir été ensevelis vivants dans les tranchées par les bulldozers américains. Les seules images qui furent proposées furent celles des milliers de combattants se rendant aux forces alliées, après la défaite. . La mise en scène. Elle a été recherchée systématiquement par les acteurs du conflit. Ainsi, l'adversaire a-t-il été stigmatisé comme la brute, celui qui recourt de façon illégitime à la violence. Les catégories de jugement manichéennes du bon et du mauvais se sont plaquées sur les présentations médiatiques de la guerre. Saddam Hussein, naguère présenté comme le rempart contre l'invasion du chiisme iranien, est devenu en quelques semaines le "bourreau ou boucher de Bagdad", ou un "nouvel Hitler", l'annexion du Koweit devenant un "Anschluss" (selon un titre de VSD). La coalition des pays alliés est rapidement devenue celle des "pays civilisés". Didier Bigo met justement en relation ce processus de stéréotypisation de l'ennemi irakien avec la disparition de la menace à l'Est. Il fallait, face à la perte des repères de la guerre froide, retrouver immédiatement un ennemi qui redonne une identité et justifie le prolongement des programmes militaires ${ }^{17}$. 


\section{LES NOUVEAUX DEFIS DE LA GESTION MEDIATIQUE DE LA VIOLENCE}

7 Pour élargir notre propos, la question de la gestion de la violence par les médias et les acteurs politiques, pose comme enjeu la capacité à contrôler des mécanismes de communication qui se sont mondialisés. Dans le cas présent, l'un des facteurs d'efficacité du contrôle de la violence dans les médias, tient à l'involontaire connivence de celui-ci avec la stratégie médiatique suivie par Saddam Hussein. On peut en effet s'étonner de la décision prise par Saddam Hussein d'expulser les journalistes occidentaux de Bagdad. Bien sûr leurs reportages de destruction auraient pu atteindre le moral et la fierté des peuples arabes mais ils auraient surtout été à même de choquer les opinions occidentales. Au début du conflit, les seules images de violence en provenance d'Irak furent celles des pilotes occidentaux faits prisonniers après des raids aériens. Ces images loin de décourager les populations occidentales ont, au contraire, éveillé un sentiment de vengeance, justifiant un peu plus l'intervention alliée. Les gouvernants se trouvent désormais dans une situation où il doivent choisir avec qui communiquer, non pas en variant les supports ou les moments, comme ce fut le cas jusqu'à présent, mais au même moment et sur le même support. On touche ici du doigt la difficulté à maîtriser des processus actuels de communication qui se sont mondialisés. Impossible désormais pour un pays de se contenter d'une communication interne, destinée à son public pour produire un effet particulier. Toute image est désormais susceptible d'être reprise par des pays de culture différente contre qui le message est adressé ; une chaîne mondiale comme CNN pouvant aller jusqu'à imposer sa sélection et sa vision des faits à l'ensemble des chaînes. Le double jeu est désormais interdit. Saddam Hussein a d'abord considéré que sa communication, ou sa noncommunication, devait être destinée aux seuls peuples arabes. Il pensait trouver en eux sa planche de salut en "s'efforçant de reprendre à son compte les bénéfices d'une stratégie contestataire en tentant de la déplacer sur la scène internationale", en acquérant l'image d'un tribun du monde arabe et musulman ${ }^{18}$. C'est pourquoi les premières images de destructions en Irak ne comprennent jamais d'images de blessés ou de morts. L'affaire du bunker de Bagdad, abri civil ou centre de commandement, marqua un changement dans la stratégie médiatique de l'Irak, et la polémique qui s'en suivit dans le monde, montra à quel point Saddam Hussein avait sans doute eu tort de ne pas user de cette arme auparavant; surtout si l'on compare les quelques rares images des bombardements sur Bagdad, semblant très ciblés et peu destructeurs, aux images abondantes et traditionnelles de dévastations occasionnées par les Scud, pourtant peu meurtriers, tombés sur Tel Aviv. Gymnases et maisons détruites, foule en pleurs, blessés sanguinolents, tous ces éléments, présents à une échelle bien plus importante en Irak, n'ont été mis en valeur qu'en Israël afin de susciter adhésion et empathie. C'est ce que font aujourd'hui les Bosniaques qui protègent coûte que coûte leurs tours émettrices, afin d'envoyer chaque jour sur les réseaux internationaux les images des atrocités commises, espérant ainsi obtenir une intervention militaire internationale. Ainsi, les journalistes ont pu parler des "redoutables Scud" et des "nuits tragiques" en Israël alors que l'Irak était simplement "bombardé", sans autres commentaires. Les images diffusées Israël jouaient toujours sur le registre émotionnel et humain tandis que les premières venues d'Irak ne présentaient que des bâtiments détruits et des foules en colère. En Israël, la violence s'est incarnée dans des individus concrets : les blessés, mais aussi Isaac Stern jouant seul du violon devant un public 
portant les masques à gaz,... ces mariés tenant des masques à la main,... les pièces étanches de chaque appartement. Ce n'est qu'à partir du bombardement du bunkerabri que l'Irak a laissé filmer ses morts et ses blessés et s'est ainsi attiré l'empathie d'une partie de l'opinion occidentale. Irakiens ou Américains, chacun croyait donc avoir intérêt à ne pas montrer ses morts : les alliés, pour ne pas rendre cette guerre impopulaire; les Irakiens, pour prouver aux peuples arabes que si les installations matérielles étaient détruites, la population, elle, continuait le "djihad". Seule la télévision irakienne aurait pu être une source de diffusion d'images de morts alliés comme le prouve son empressement à diffuser des images des pilotes capturés - mais elle n'en n'eut ni l'occasion ni les moyens. Et lorsque l'Irak eut changé de stratégie, en rétablissant les autorisations de tournage, il a tout de même continué à se discréditer en encadrant les journalistes et en ne leur laissant pas filmer ce qu'ils voulaient, jetant un voile de suspicion sur toute image de destruction ou de souffrance. La censure israélienne, par contre, a réussi à se faire reconnaître comme juste et limitée, en faisant passer son action comme la simple préoccupation d'interdire à l'ennemi de repérer géographiquement le lieu d'impact, et ajuster son tir. Pour finir, nous pourrions dire que la gestion médiatique de la violence est devenue un enjeu de la lutte politique entre les nations. En ce sens, la guerre du Golfe est une excellente illustration de la théorie du "soft power" qui place la maitrise des moyens de communication et du contenu de ceux-ci au rang d'éléments de la puissance internationale ${ }^{19}$. Notamment lorsqu'il s'agit de canaliser des phénomènes déstabilisants comme les actes de violence politique.

\section{NOTES}

1. Sur les libertés dont disposèrent les journalistes au Vietnam voir Hallin, D.C., The "Uncensored War" : the Media and Vietnam, New-York-Oxford, Oxford University Press, 1986.

2. Il ne s'agit toutefois pas ici de faire passer l'analyse de la violence au second plan par rapport aux logiques de fonctionnement des médias. Sur l'action des médias durant la Guerre du Golfe de nombreux ouvrages ont été écrits, se reporter notamment à Wolton (D.), War Game : l'Information et la Guerre, Paris, Flammarion, 1991 ; Médiaspouvoirs : "Les Médias dans la Guerre.", n²3, juillet-septembre 1991, Paris, Bayard Presse. Leblanc (G.), "Sur la Terre comme au Ciel", L'Image Vidéo, n 8 , mars-avril 1991, pp. 16-23. Ferro (M.), L'Information en Uniforme, Paris, Ramsay, 1991, 121p ; Zelizer (B.), "CNN, the Gulf War and Journalistic Practice, "Journal of Communication, vol.42, n 1 , hiver 1992, pp. 66-81.

3. Notre analyse peut toutefois être contestée, certains militaires ou politiques affirmant qu'il existe des "guerres justes" conformes aux idéaux démocratiques. Pour une synthèse sur ces questions voir Walzer (M.), Just and Unjust Wars. A Moral Argument with Historical Illustrations, New-York, Basic Books, 1977, 361p.

4. « Les notions essentielles de cette théorie sont extrêmement simples : l'existence simultanée d'éléments de connaissance qui d'une manière ou d'une autre ne s'accordent pas (dissonance) entraîne de la part de l'individu un effort pour les faire 
d'une façon ou d'une autre mieux s'accorder (réduction de la dissonance) ». Festinger (L.), Aronson (E.) "Eveil et Réduction de la Dissonance dans des contextes sociaux." in Levy (A.), Psychologie Sociale. Textes Fondamentaux, Paris, Dunod, 1977 (1ère édition 1965) pp.193-211.

5. Un géopoliticien comme Gérard Chaliand partage lui aussi cet avis, prouvant, par là même, combien l'idée est répandue dans les milieux militaires. Il se déclare favorable à la censure car : «L'arrière est un enjeu des guerres modernes. Il n'est pas acceptable de donner dans la guerre-spectacle, strictement émotive, où l'arrière finit par compter plus que le front lui-même. Les guerres aujourd'hui, se déroulent essentiellement dans les esprits et les volontés ", Interview à Télérama, n²141, 23 janvier 1991, p.21.

6. Sur les rapports entre les militaires et les médias en situation de guerre, se reporter notamment à : Hammond (W.M.), Public Affairs : the Military and the Media, 1962-1968, Washington DC, Center of Military History United States Army, 1988 ; Harris (R.)., Gotcha! The Media, the Government and the Falklands Crisis, Londres, Faber \& Faber, 1983 ; Hooper (A.), The Military and the Media, Aldershot, Gower, 1982.

7. Hacker (F.), Agression, Violence dans le monde moderne, Paris, Calmann-Lévy, 1972, p.113.

8. Hermant (D.), "Les discours, la menace, l'ordre international et la guerre du Golfe", Stratégique, 1992 (2), n54, p.173.

9. Cf. sur ce point et sur l'importance de la fonction scopique assumée par les médias, l'article de Dufour-Gompers (R Y.), "Voir la violence de la guerre ou le "théâtre des opérations", Revue Internationale des Sciences Sociales, n¹32, mai 1992, pp. 249-268. 10. Dans ce passage, Frédéric Bon recense un certain nombre d'euphémismes dont "la politique militaire abonde" : «La bombe atomique [devient] force de frappe, ou mieux force de dissuasion. La [guerre] coloniale s'habille du terme de pacification. Les corps expéditionnaires deviennent conseillers techniques, les unités d'élite se métamorphosent en soldat de la paix ». Bon (F.), "Langage et Politique" in Leca et Grawitz., Traité de Science Politique, vol.3, Paris, PUF, 1985. Repris in Les Discours de la Politique, Paris, Economica, 1991, p.274.

11. Michel Chevallet, spécialiste de la rubrique scientifique à TF1, annonce quant à lui dans le $20 \mathrm{~h}$. du 21 janvier que : «Quelle que soit l'issue de ce combat, le grand vainqueur en sera la technologie».

12. "Opération Koweit", VSD, numéro Hors Série, hiver 1990, p.1, p.43.

13. Interview à L'Evénement du Jeudi, le 21/02/1991.

14. Foucault (M), Surveiller et Punir, naissance de la prison, Paris, Gallimard, 1975.

15. Ferro, (M.), L'Information en Uniforme. Paris, Ramsay, 1991, p.22.

16. Pierre Babey, FR3, "19/20", 23 janvier 1991.

17. Bigo (D.), in Approches Polémologiques. Conflits et violence dans le monde au tournant des années 90, Paris, FEDN, 1991, p.42.

18. Bertrand Badie développe l'hypothèse selon laquelle la victoire militaire n'était pas un but recherché en soi par Saddam Hussein. Pour lui "seul compte le niveau de mobilisation atteint". Une logique tribunitienne prévalait, tendant à imposer l'image de tribun du monde arabe, du monde musulman, "voire des déshérités du monde entier face à la puissance occidentale." ; Badie (B.), "La Guerre Contestataire", Revue du Monde Musulman et de la Méditerranée, numéro Hors Série, 1991, p. 54-56.

19. Voir notamment Nye (J.), S. "Soft Power", Foreign Policy, n80, automne 1990, pp. 153-171 ; Strange (S.), States and Markets, Londres, Pinter Publishers, 1988. 


\section{RÉSUMÉS}

Les images de violence et de destruction ont été le plus possible cachées par la censure militaire. La violence n'a cependant pas totalement été masquée. Elle a plutôt été retravaillé, représentée, en passant à travers le prisme télévisuel et journalistique. Elle a été montrée mais euphémisée, mise en scène ou esthétisée. Ce travail sur l'image n'est pas uniquement le fruit de la censure mais aussi des logiques médiatiques et journalistiques à l'oeuvre lors de la guerre du Golfe. Il convient donc de mettre au jour ces logiques de traitement de la violence, en s'interrogeant sur les responsabilités respectives des deux principales catégories d'acteurs : militaires et journalistes. Cette recherche pose plus largement la question des stratégies d'utilisation d'un outil transnational comme la télévision. En effet, la gestion de la violence dans le cadre d'une communication politique nationale est désormais problématique, dans un univers médiatique mondialisé. Ainsi Irakiens et Américains, ont fait chacun le choix de ne pas montrer les morts. Option qui fut sans doute une "erreur" stratégique pour Saddam Hussein et qu'il modifia trop tard.

\section{INDEX}

Mots-clés : guerre, médias, violence, visuel

Index géographique : Irak, Moyen-Orient

Index chronologique : 1991 